\title{
Perceived Efficacy of COVID-19 Prevention Measures and Intention to Use Among Chronic Disease Patients in Northeast Ethiopia, 2020
}

Abebe Dires ( $\sim$ abede9907@gmail.com )

Wollo University https://orcid.org/0000-0002-0186-1123

Sisay Gedamu Addis

Wollo University

Yemiamrew Getachew Tesema

Wollo University

Research article

Keywords: Perception, efficacy, COVID-19 prevention measures, chronic disease, Ethiopia

Posted Date: September 25th, 2020

DOl: https://doi.org/10.21203/rs.3.rs-73649/v1

License: (c) (i) This work is licensed under a Creative Commons Attribution 4.0 International License.

Read Full License 


\section{Abstract}

Background: The novel corona virus disease outbreak which was first detected in China is caused by sever acute respiratory syndrome corona virus 2. In Ethiopia, poor perception about COVID-19 prevention methods might be one of determinant factor to the low utilization of adopted prevention measures and increasing of cases. However, it was unknown to what extent individuals were perceived the efficacy of those measures. Hence, this study was aimed to assess perceived efficacy of COVID-19 preventive measures and intention to carry out those methods among chronic disease patients.

Methods: Institutional based cross sectional study was conducted among 413 chronic disease patients from 21 July - 5 August, 2020. A pre-tested interviewer administered structured questionnaire was used for data collection. Both binary and multivariable logistic regression analyses were used and in multivariable analysis, variables were declared statistically significant at $p$-value of $<0.05$.

Results: In overall, $42.1 \%$ of participants had low perception to the efficacy of COVID-19 prevention measures and $28.3 \%$ had low intention to carry out those prevention measures. In this study, young adults (AOR=2.48; 95\% Cl: 1.42-4.31), male gender (AOR=2.75; 95\% Cl: 1.73-4.37), uneducated ( $\mathrm{AOR}=3.42$; $95 \% \mathrm{Cl}: 1.47-7.94)$ and face mask non-users ( $\mathrm{AOR}=1.64 ; 95 \% \mathrm{Cl}: 1.03-2.61)$ were significantly associated with low perceived efficacy of COVID-19 prevention methods. However, male gender (AOR=2.06; 95\% Cl: 1.28-3.31), rural residents (AOR=1.89; $95 \% \mathrm{Cl}$ : 1.16-3.07) and patients with longer duration of chronic disease (AOR= 2.19; 95\% Cl: 1.19-4.01) were significantly associated with low intention to carry out COVID-19 prevention methods.

Conclusion: In this study, significant proportion of patients (42\%) had low perception about the efficacy of COVID-19 prevention measures and nearly one-third of participants (28.3\%) had low intention to carry out those measures. Thus, interventions aimed to enhance patients' attitude and behavioral changes on COVID-19 prevention measures have to be provided.

\section{Background}

The 2019 Novel Corona Virus Disease (COVID-19) was caused by the Sever Acute Respiratory Syndrome Corona Virus 2 (SARS-CoV-2) and World Health Organization (WHO) declared it as a public health emergency of global concern on 30 January (1) and a pandemic disease on 11 march 2020 (2). COVID19 has been distributed nearly in all countries and it was estimated that about 349 million peoples are at risk of severe COVID-19 (3). Globally, as of August 16, 2020 a total of 21.2 million confirmed cases of COVID-19 and 761 thousand deaths were reported and America and South East Asia were the most affected regions (4). In Africa, the distribution of COVID-19 cases was not as high as estimated $(5,6)$. However, South Africa, Nigeria, Niger and Ethiopia were among highly burdened countries by COVID-19 pandemic in the continent (4). In Ethiopia, the first confirmed case of COVID-19 was reported in 13 March, 2020 (7). Since then, the number of peoples infected with the virus have been increasing gradually from March to April (8). In Ethiopia, from 10-16 August 2020, the number of cases and death due to COVID-19 
was increased by $56 \%$ and $12 \%$, respectively; Despite of the decreased number of newly reported cases and death in Africa (4). In Ethiopia, the number of infected people is expected to be high in the community due to the fact that limited COVID-19 testing sites are available in the country. As of 30 August, 2020, a total of 50,000 peoples have been infected with corona virus and 770 deaths were reported in Ethiopia (9).

Overall, case underestimation is a common problem in African countries where lack of resources and poor infrastructure were the main determinant factors (10).

COVID-19 is a highly infectious disease transmitted from infected persons to other susceptible group of individuals by respiratory droplets through coughing, sneezing, and breathing $(11,12)$. Contaminated materials with nasopharyngeal secretion can transmit the virus if get access to the nasal and buccal mucosa and the eye (12). All infected patients may not show symptoms of the disease (13). Majority of infected patients were presented with mild symptoms of pneumonia like fever, cough, fatigue and dyspnea (14-16). However, small proportion of cases could develop severe form of the disease like acute respiratory distress syndrome, organ dysfunction and death $(17,18)$. Every individual is expected to be susceptible for the novel corona virus infection. However, studies revealed that severity of COVID-19 was found to be high in advanced age and patients who had chronic disease concomitantly $(11,16,18,19)$.

Cardiovascular, diabetic mellitus and chronic lung diseases were the main medical conditions associated with COVID-19 illness and death (20). In southern and eastern Africa, more than 20.7 million peoples have been living with Human Immuno-deficiency Virus (HIV) in 2019 (21). In Ethiopia, the burden of chronic diseases, particularly cardiovascular disease have been increased for the last decades (22). It is also one of African country with high burden of TB and TB/HIV co-infection (23). This double burden of chronic communicable and non-communicable disease in the country may contribute for the severe clinical illness and mortality of COVID-19 unless prevention methods are applied.

As of august 2020, there was no effective anti-viral drug to cure COVID-19 globally which was approved by WHO (4). Thus, patients with COVID-19 are being treated symptomatically and supportive measures have been provided based on recent guidelines (24). However, the type of supportive measures and clinical setups used for monitoring cases was varied among countries and it mainly depends on their economical status (25). Starting from the onset of COVID-19 epidemic, countries' economical, social and political issues were greatly influenced (26-31). Moreover, it brought a significant impact on health care system of Africa including Ethiopia (32-34). In Ethiopia, this pandemic has also brought a major socioeconomical impact $(35,36)$. A study done in Addis Ababa revealed as only $10.3 \%$ of households can meet their food for more than 1 month with their current saving (37). Currently, individuals who didn't follow WHO and national recommendations of COVID-19 prevention and control methods are at risk of the disease. Lack of epidemiological data, mistrust and political issues were the main factors that hinder the response against the disease particularly in east Africa (38). In Ethiopia, the number of cases has been increased dramatically after first July, 2020. The main reason could be related to the mass demonstration and political instability in Addis Ababa and some towns in Oromia region. 
Developing countries faced a great challenge in providing critical care and support to COVID-19 cases (39). Thus, primary prevention strategies are feasible and the best options in resource limited countries (40). A person can be infected after contact history with a known corona virus infected person or from any other unknown source of infection (41). Thus, maintaining physical distance, washing hands with soap and water, staying home, wear face mask and seeking medical advice were the main WHO recommended behaviors to prevent the virus and Ethiopia also adopted those strategies (42). Studies revealed that timely and effectively implemented physical and social distance alone can reduce COVID-19 distribution by $60-95 \%$ (13). Few community based studies conducted in Ethiopia in different segment of the population revealed that majority of the urban population had good knowledge about COVID-19 and its prevention methods (43-46). However, maintaining of COVID-19 prevention methods and behaviors in the community was not satisfactory even if the disease is fatal and wide spreading all over the country (47). Special attention should be provided for chronic disease patients due to their high risk of death. In Ethiopia, the number of peoples infected with novel corona virus have been increasing dramatically from day to day despite of government prevention activities (8). Low perception of peoples about their susceptibility to COVID-19 and its severity were the major predictor to the low utilization and engagement of COVID-19 preventive behaviors $(48,49)$. Poor perception of COVID-19 prevention methods and behaviors might be also one of the main determinant factors to the low utilization of adopted mitigation measures and increasing of cases in Ethiopia. Hence, this study was aimed to assess perceived efficacy of COVID-19 preventive measures and intention to use them among chronic disease patients.

\section{Methods And Materials}

\section{Study design, period and setting}

Institutional based cross sectional study was conducted among chronic disease patients in Hospitals of Dessie town, northeast Ethiopia from 21 July - 5 August, 2020. Dessie town is located 400 kilometer far from Addis Ababa (capital city of Ethiopia) and $488 \mathrm{~km}$ from Bahirdar (capital city of Amhara regional government). It is located at an altitude of 2470 meter above sea level and based on the 2007 national census, the town has a total population of 151,094, of which 78,203 are females (50). In the town, two governmental and three private hospitals are available that have been serving populations of Dessie town and surrounding zones. In addition, both COVID-19 quarantine and treatment centers are available in the town.

\section{Population}

All chronic disease patients who were admitted or attended chronic disease follow up units in hospitals of Dessie town were the source population.

Whereas, all patients who were admitted or attended in selected hospitals of Dessie town during data collection period were the study population.

\section{Sample size determination}


A single population proportion formula $\left[n=(Z \text { a/2 })^{2} P(1-P) / d^{2}\right]$ was used to estimate the sample size. Since, no previous study was conducted in Ethiopia; we have used maximum sample size assumptions. Proportion of $50 \%, 95 \%$ confidence level $(Z=1.96)$ and $5 \%$ margin of error. With this, the required sample size was 384 . By adding $10 \%$ non response rate, a total of 422 chronic disease patients were planned to be involved in the study.

\section{Sampling technique and procedures}

From five hospitals in the town, one government hospital (Dessie referral hospital) and two private hospitals (Ethio general hospitals and Selam general hospitals) were selected using lottery method. Then, the daily average chronic disease patients attended those hospitals at both out-patient clinics and inpatient units were estimated. Finally, based on their patients load, sample was allocated proportionally in the three hospitals: $\mathrm{DRH}=295, \mathrm{EGH}=67, \mathrm{SGH}=60$. Finally, data was collected from eligible patients by using systematic random sampling technique.

\section{Data collection tool and procedures}

The data collection tool which includes socio-demographic characteristics, clinical profile and health seeking behaviors of patients was adopted by reviewing different literatures (51-53). However, patients' perceived efficacy of COVID-19 prevention measures and their intention to carry out those measures was adopted from a standard tool on effective communication in outbreak management for Europe (54). A pre-tested interviewer administered questionnaire was used to collect the data. However, patients' chart was also reviewed to identify the type of chronic disease and presence of other co-morbidities that they had. Moreover, patients were observed whether or not they have used face mask and hand sanitizers at the time of data collection.

Perception of patients to the efficacy of COVID-19 prevention methods and their intention to carry out those prevention methods was assessed by six questions (for both efficacy and intention to carry out measures) in which, each question had a five scale responses; ( $1=$ certainly not, $2=$ probably not, $3=$ perhaps not-perhaps yes, $4=$ probably yes and $5=$ most certainly).

For the purpose of analysis; those who responded "certainly not", "probably not" and "perhaps notperhaps yes" was coded by "0" and those who responded "probably yes" and "most certainly" was coded by " 1 " for each question. Finally, their score out of six was determined and those who scored "0-3" were considered as having low perception and those who scored "4-6" were considered as having high perception about the efficacy of COVID-19 prevention methods. Furthermore, those who scored "0-3" were considered as having low intention to carry out COVID-19 prevention methods and those who scored "4-6" were considered as having high intention to carry out those prevention measures. Finally, the main hindering factors to carry out COVID-19 preventive measure were also assessed for those who responded "certainly not" and "probably not".

\section{Data quality control}


Data quality was insured by undertaking the following measures. The questionnaire was translated from English to Amharic (local language of the study area) by considering the culture and norms of the society and then back to English to check its consistency. Data collectors and supervisors were trained for two days regarding objective of the study, ethical issues and how to collect the data by protecting themselves from COVID-19. They were also trained how to apply personal protective equipments and glove, face mask and sanitizer were provided for both data collectors and supervisors. In addition, the tool was pretested in Bati general hospital by taking $10 \%$ of the total sample size so as to check for clarity of language and appropriateness of the tools.

\section{Data processing and Analysis}

Data was coded and entered using Epi-Data version 3.1 statistical software and then exported to SPSS (statistical package software for social science) version 20.0 for further statistical analysis. Appropriate descriptive statistics was used to analyze the data and mean, standard deviation (SD), frequency, percentage and tables were utilized to summarize the data. Binary and multivariable logistic regression analyses were tested to identify factors associated to low perceived efficacy of COVID-19 prevention measures and low intention to carry out those prevention activities. In bivariable analysis, variables which had a p-value of less than 0.2 were entered into multivariable analysis model. In multivariable analysis, variables were declared statistically significant at $p$-value of $<0.05$.

Moreover, strength of association between factors and the dependent variables were determined using Adjusted Odds Ratio (AOR) with 95\% confidence level.

\section{Results}

\section{Socio-demographic characteristics of study participants}

A total of 413 adults with chronic disease were participated in the study with response rate of $97.8 \%$. The mean age of participants was 48.2 years (SD \pm 15.8 years) and $215(52.1 \%)$ were females. Majority of participants, 268(64.9\%) were urban dwellers and 287(69.5\%) participants were married. Of all participants, $191(46.2 \%)$ had no formal education and 159(38.5\%) were housewives. The mean family size and house room number of participants was $4.4(\mathrm{SD} \pm 1.7)$ and $3.0(\mathrm{SD} \pm 1.4)$, respectively (Table 1). 
Table 1

Socio-demographic characteristics of chronic disease patients in Dessie town, northeast Ethiopia, $2020(n=413)$

\begin{tabular}{|c|c|c|c|}
\hline Variables & Category & Frequency & Percentage \\
\hline \multirow[t]{3}{*}{ Age in year } & $18-34$ & 139 & 33.7 \\
\hline & $35-54$ & 128 & 31.0 \\
\hline & $>=55$ & 146 & 35.3 \\
\hline \multirow[t]{2}{*}{ Sex } & Female & 215 & 52.1 \\
\hline & Male & 198 & 47.9 \\
\hline \multirow[t]{2}{*}{ Residence } & Urban & 268 & 64.9 \\
\hline & Rural & 145 & 35.1 \\
\hline \multirow[t]{4}{*}{ Marital status } & Single & 86 & 20.8 \\
\hline & Married & 287 & 69.5 \\
\hline & Divorced & 15 & 3.6 \\
\hline & Widowed & 25 & 6.1 \\
\hline \multirow[t]{6}{*}{ Occupation } & Housewives & 159 & 38.5 \\
\hline & Employed & 85 & 20.6 \\
\hline & Students & 61 & 14.8 \\
\hline & Farmer & 60 & 14.5 \\
\hline & Unemployed & 27 & 5.1 \\
\hline & Merchant & 21 & 6.5 \\
\hline \multirow[t]{4}{*}{ Educational status } & No formal education & 191 & 46.2 \\
\hline & Primary school & 91 & 22.0 \\
\hline & Secondary school & 87 & 21.1 \\
\hline & Tertiary and above & 44 & 10.7 \\
\hline \multirow[t]{2}{*}{ Household family size } & $1-3$ & 167 & 40.4 \\
\hline & $>=4$ & 246 & 59.6 \\
\hline \multirow[t]{3}{*}{ Household room number } & 1 & 83 & 20.1 \\
\hline & 2 & 98 & 23.7 \\
\hline & $>=3$ & 232 & 56.2 \\
\hline
\end{tabular}




\section{Clinical characteristics and risk assessment of study participants}

Of all study participants, $94(22.8 \%)$ and $86(20.8 \%)$ were hypertensive and diabetic patients, respectively. The patient's mean duration of illness was 3.7 years (SD \pm 5.6 year) and $42(10.2 \%)$ patients have been living with their disease for more than ten years. Majority of participants, 350 (84.7\%) had no additional co-morbidities; and $63.2 \%$ and $26.6 \%$ of participants were utilized face masks and sanitizer, respectively at the time of data collection. Regarding risk assessment to the current pandemic, no one had contact history with a known confirmed COVID-19 cases: However, 41(9.9\%) patients had reported respiratory symptoms and $16(3.9 \%)$ had travel history to other areas in the last two weeks. Furthermore, 194(47\%) participants were members of community based health insurance and $81(19.6 \%)$ clients will have no social support if they were isolated or quarantined due to COVID-19 (Table 2). 
Table 2

Clinical characteristics, health seeking behavior and risk assessment of chronic disease patients in Dessie town, northeast Ethiopia, $2020(n=413)$

\begin{tabular}{|c|c|c|c|}
\hline Variables & Category & Frequency & $\begin{array}{l}\text { Percentage } \\
(\%)\end{array}$ \\
\hline \multirow[t]{8}{*}{ Type of chronic disease } & Hypertension & 94 & 22.8 \\
\hline & $\begin{array}{l}\text { Diabetes } \\
\text { mellitus }\end{array}$ & 86 & 20.8 \\
\hline & Heart disease & 76 & 18.4 \\
\hline & $\begin{array}{l}\text { kidney } \\
\text { disease }\end{array}$ & 35 & 8.5 \\
\hline & $\begin{array}{l}\text { respiratory } \\
\text { disease }\end{array}$ & 30 & 7.3 \\
\hline & HIV/AIDS & 22 & 5.3 \\
\hline & $\begin{array}{l}\text { Multiple } \\
\text { illness * }\end{array}$ & 63 & 15.3 \\
\hline & Others\$ & 7 & 1.7 \\
\hline \multirow[t]{3}{*}{ Duration of chronic disease( in year) } & $<5$ & 307 & 74.3 \\
\hline & $5-10$ & 64 & 15.5 \\
\hline & $>10$ & 42 & 10.2 \\
\hline \multirow[t]{2}{*}{ Presence of additional Co-morbidity? } & No & 350 & 84.7 \\
\hline & Yes & 63 & 15.3 \\
\hline \multirow[t]{2}{*}{ Presence of respiratory symptoms in the last 2 weeks? } & No & 372 & 90.1 \\
\hline & Yes & 41 & 9.9 \\
\hline \multirow[t]{2}{*}{ Travel history to other areas in the last 2 weeks? } & No & 397 & 96.1 \\
\hline & Yes & 16 & 3.9 \\
\hline \multirow[t]{2}{*}{ Are you member of community based health insurance? } & No & 219 & 53.0 \\
\hline & Yes & 194 & 47.0 \\
\hline \multirow{2}{*}{$\begin{array}{l}\text { Do you have social support if you are isolated/ } \\
\text { quarantined due to COVID-19? }\end{array}$} & Yes & 332 & 80.4 \\
\hline & No & 81 & 19.6 \\
\hline Have you wear face mask? & Yes & 261 & 63.2 \\
\hline
\end{tabular}




\begin{tabular}{|llll|}
\hline Variables & Category & Frequency & $\begin{array}{l}\text { Percentage } \\
\text { (\%) }\end{array}$ \\
\hline & No & 152 & 36.8 \\
\hline Do you use hand sanitizers? & No & 303 & 73.4 \\
\cline { 2 - 4 } & Yes & 110 & 26.6 \\
\hline SNerve disease, cancer thad more than one chronic disease & & & \\
\hline
\end{tabular}

\section{Perceived efficacy of COVID-19 prevention methods, intention to carry out measures and barriers}

In this study, 155(37.5\%) participants were perceived as applying face masks "probably" helps to prevent COVID-19 and 185(44.8\%) were perceived as hand washing with soap and water "probably" helps to prevent COVID-19. Whereas, $21.3 \%$ and $20.6 \%$ of respondents perceived that maintaining of physical distancing and wearing face mask may "not probably" helps to prevent the current pandemic disease, respectively (Table 3). In overall, $174(42.1 \%)(95 \%$ Cl: $37.3-46.5)$ participants had low perception to the efficacy of COVID-19 prevention measures and 117(28.3\%) (95\% Cl: 24.1-32.7) participants had low intention to carry out those prevention measures. Specifically, $85.5 \%, 81.9 \%$ and $74.6 \%$ of participants had an intention to carry out hand washing, wearing face mask and will maintain physical distance, respectively (Table 3 ).

In this study, we have also assessed the main reason why participants had no willingness (for those who responded "certainly not "and "probably not") to carry out the most important recommended COVID-19 prevention measures and behaviors. Thus, takes too much effort was the main reason identified by participants for not willing to wear face masks (38.6\%), wash their hands with soap and water $(43.7 \%)$ and not to use alcohol based hand sanitizers (67.8\%). In addition, $33.8 \%$ of participants described that they will not maintain physical distancing because they had doubt whether it helps to prevent the disease or not. Moreover, about $24.8 \%$ of participants responded that they will not stay home so as to prevent COVID-19 because of other peoples in their environment would not carry out this measure (Table 4). 


\section{Number (\%)}

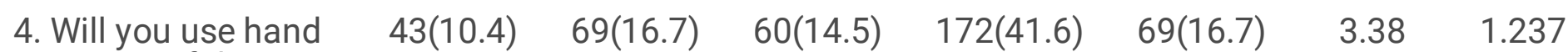
sanitizers, if this is advised?

5 . Will you staying home? If this is $35(8.5)$ $86(20.8)$

65(15.7)

205(49.6)

22(5.3)

3.23

1.099 advised?

6. Will you quarantine yourself, 13(3.1) 26(6.3) 11(2.7) 287(69.5) 76(18.4) 3.94 0.862 if you are suspected and advised to do it?

Note: level 1 = certainly not, level 2 = probably not, level 3 = perhaps not-perhaps yes, level 4 = probably yes, level $5=$ most certainly

Table 4

Main hindering factors not to willing to carry out COVID-19 preventive measure among chronic disease patients in Dessie town, northeast Ethiopia, $2020(n=413)$

\section{Prevention methods}

\section{Main reason, number (\%)}

$\begin{array}{llllll}1 & 2 & 3 & 4 & 5 & 6\end{array}$

1. Why you are not willing to use face masks?

$\begin{array}{llllll}6(10.5) & 22(38.6) & 4(7.0) & 13(22.8) & 9(15.8) & 3(5.3)\end{array}$

2. Why you are not willing to maintain Physical distancing?

$\begin{array}{lllll}11(13.7) & 7(8.7) & 19(23.8) & 27(33.8) & 16(20.0)\end{array}$

3. Why you are not willing to wash your hands with soap

$8(16.7) \quad 21(43.7) \quad 5(10.4) \quad 14(29.2)$
and water?

4. Why you are not willing to use hand sanitizers?

5. Why you are not willing to stay home?

6. Why you are not willing to quarantine yourself if you are suspected?
Legend: 1 = COVID-19 is not serious, 2 = Takes too much effort (time, resource), $3=$ I do not think I am at risk of contracting COVID-19, 4 = I doubt whether the measures help, 5 = People in my environment will also not carry out the measure, 6 = others

Factors associated with low perceived efficacy of COVID-19 prevention methods and intention to carry out measures.

In bivariable analysis; age, sex, residence, educational level, duration of illness, presence of additional comorbidity, respiratory symptoms, social support, COVID-19 prevention behaviors(facemask and sanitizer 
utilization) had a p-value of $<0.2$ ( Table.5) and were entered into multivariable logistic regression analysis to identify independent predictors. In multivariable analysis; young adults (AOR $=2.48 ; 95 \% \mathrm{Cl}$ : 1.42-4.31), male gender $(\mathrm{AOR}=2.75 ; 95 \% \mathrm{Cl}: 1.73-4.37)$, uneducated $(\mathrm{AOR}=3.42 ; 95 \% \mathrm{Cl}: 1.47-7.94)$ and face mask non-users $(A O R=1.64 ; 95 \% \mathrm{Cl}: 1.03-2.61)$ were significantly associated with low perceived efficacy of COVID-19 prevention methods and behaviors. Furthermore, male gender (AOR = 2.06; $95 \% \mathrm{Cl}: 1.28-3.31)$, rural residents ( $\mathrm{AOR}=1.89 ; 95 \% \mathrm{Cl}: 1.16-3.07)$ and patients with longer duration of illness (AOR $=2.19 ; 95 \% \mathrm{Cl}: 1.19-4.01),(\mathrm{AOR}=2.40 ; 95 \% \mathrm{Cl}: 1.15-4.98)$ were significantly associated with low intention to carry out COVID-19 prevention methods and behaviors (Table 6). 
Table 5

Proportion of perceived efficacy and intention to carry out COVID-19 prevention methods among chronic disease patients in Dessie town, northeast Ethiopia, $2020(n=413)$

\begin{tabular}{|c|c|c|c|c|c|c|}
\hline \multirow[t]{2}{*}{ Characteristics } & \multicolumn{2}{|c|}{$\begin{array}{l}\text { Perceived efficacy of } \\
\text { COVID-19 prevention } \\
\text { methods }\end{array}$} & \multirow[t]{2}{*}{ P-value } & \multicolumn{2}{|c|}{$\begin{array}{l}\text { Intention to carry out } \\
\text { CoVID- } 19 \text { prevention } \\
\text { methods }\end{array}$} & \multirow[t]{2}{*}{$\begin{array}{l}\mathrm{P}- \\
\text { value }\end{array}$} \\
\hline & $\begin{array}{l}\text { High }(\mathrm{N}= \\
239) \text {, } \\
\mathrm{n}(\%)\end{array}$ & $\begin{array}{l}\text { Low(N = } \\
174), n(\%)\end{array}$ & & $\begin{array}{l}\operatorname{High}(\mathrm{N}= \\
296), \mathrm{n} \\
(\%)\end{array}$ & $\begin{array}{l}\operatorname{Low}(N=117), n \\
(\%)\end{array}$ & \\
\hline Age( year) & & & 0.018 & & & 0.182 \\
\hline $18-34$ & $67(48.2)$ & $72(51.8)$ & & $95(68.3)$ & $44(31.7)$ & \\
\hline $35-54$ & $79(61.7)$ & 49(38.3) & & $91(71.1)$ & $37(28.9)$ & \\
\hline$>=55$ & $93(63.7)$ & $53(36.3)$ & & $110(75.3)$ & $36(27.4)$ & \\
\hline Sex & & & $<.001$ & & & 0.005 \\
\hline Male & $97(49.0)$ & $101(51.0)$ & & $129(65.2)$ & $69(34.8)$ & \\
\hline Female & $142(66.0)$ & 73(34.0) & & 167(77.7) & $48(22.3)$ & \\
\hline Residence & & & 0.002 & & & 0.001 \\
\hline Urban & $170(63.4)$ & $98(36.6)$ & & 207(77.2) & $61(22.8)$ & \\
\hline Rural & $69(47.6)$ & $76(52.2)$ & & $89(61.4)$ & $56(38.6)$ & \\
\hline Educational status & & & 0.006 & & & 0.159 \\
\hline No formal education & $95(49.7)$ & $96(50.3)$ & & $134(70.2)$ & $57(29.8)$ & \\
\hline Primary school & $53(58.2)$ & $38(41.8)$ & & $63(69.2)$ & $28(30.8)$ & \\
\hline Secondary school & $59(67.8)$ & $28(32.2)$ & & $63(72.4)$ & $24(27.6)$ & \\
\hline College and above & $32(72.7)$ & $12(27.3)$ & & $36(81.8)$ & $8(18.2)$ & \\
\hline Marital status & & & 0.407 & & & 0.812 \\
\hline Single & $54(62.8)$ & $32(37.2)$ & & $65(75.6)$ & $21(24.4)$ & \\
\hline Married & $166(57.8)$ & $121(42.2)$ & & 203(70.7) & $84(29.3)$ & \\
\hline Divorced & 8(53.3) & $7(46.7)$ & & $10(66.7)$ & $5(33.3)$ & \\
\hline Widowed & $11(44.0)$ & $14(56.0)$ & & $18(72.0)$ & $7(28.0)$ & \\
\hline $\begin{array}{l}\text { Type of chronic } \\
\text { disease }\end{array}$ & & & 0.516 & & & 0.277 \\
\hline Diabetes mellitus & $53(61.6)$ & $33(38.4)$ & & 63(73.3) & $23(26.7)$ & \\
\hline
\end{tabular}




\begin{tabular}{|c|c|c|c|c|c|c|}
\hline \multirow[t]{2}{*}{ Characteristics } & \multicolumn{2}{|c|}{$\begin{array}{l}\text { Perceived efficacy of } \\
\text { COVID-19 prevention } \\
\text { methods }\end{array}$} & \multirow[t]{2}{*}{ P-value } & \multicolumn{2}{|c|}{$\begin{array}{l}\text { Intention to carry out } \\
\text { COVID-19 prevention } \\
\text { methods }\end{array}$} & \multirow[t]{2}{*}{$\begin{array}{l}\mathrm{P} \text { - } \\
\text { value }\end{array}$} \\
\hline & $\begin{array}{l}\text { High }(\mathrm{N}= \\
239), \\
\text { n (\%) }\end{array}$ & $\begin{array}{l}\text { Low(N= } \\
174), n(\%)\end{array}$ & & $\begin{array}{l}\operatorname{High}(\mathrm{N}= \\
296), \mathrm{n} \\
(\%)\end{array}$ & $\begin{array}{l}\operatorname{Low}(\mathrm{N}=117), \mathrm{n} \\
(\%)\end{array}$ & \\
\hline Hypertension & $56(59.6)$ & $38(40.4)$ & & 67(71.3) & $27(28.7)$ & \\
\hline kidney disease & $21(60.0)$ & $14(40.0)$ & & 29(82.9) & $6(17.1)$ & \\
\hline Heart disease & $46(60.5)$ & $30(39.5)$ & & $53(69.7)$ & $23(30.3)$ & \\
\hline Respiratory disease & 11(36.7) & 19(63.3) & & $16(53.3)$ & $14(46.7)$ & \\
\hline HIV/AIDS & $12(54.7)$ & $10(45.5)$ & & $14(63.6)$ & $8(36.4)$ & \\
\hline Multiple illness & $36(57.1)$ & $27(42.9)$ & & $50(79.4)$ & 13(20.6) & \\
\hline Others & $4(57.1)$ & $3(42.9)$ & & $4(57.1)$ & $3(42.9)$ & \\
\hline Family size & & & 0.297 & & & 0.238 \\
\hline $1-3$ & 103(61.7) & $64(38.3)$ & & $125(74.9)$ & $42(25.1)$ & \\
\hline$>=4$ & $136(55.3)$ & $110(44.7)$ & & $171(69.5)$ & $75(30.5)$ & \\
\hline Houseroom number & & & 0.868 & & & 0.573 \\
\hline 1 & $46(55.4)$ & $37(44.6)$ & & $56(67.5)$ & $27(32.5)$ & \\
\hline 2 & $58(59.2)$ & $40(40.8)$ & & 73(74.5) & $25(25.5)$ & \\
\hline$>=3$ & $135(58.2)$ & $97(41.8)$ & & $167(72.0)$ & $65(28.0)$ & \\
\hline $\begin{array}{l}\text { Duration of illness ( } \\
\text { year) }\end{array}$ & & & 0.153 & & & 0.025 \\
\hline$<5$ & $186(60.6)$ & $121(39.4)$ & & $231(75.2)$ & $76(24.8)$ & \\
\hline $5-10$ & $33(51.6)$ & $31(48.4)$ & & $39(60.9)$ & 25(39.1) & \\
\hline$>10$ & $20(47.6)$ & $22(52.4)$ & & 26(61.9) & 16(38.1) & \\
\hline $\begin{array}{l}\text { Presence of other co- } \\
\text { morbidity }\end{array}$ & & & 0.126 & & & 0.041 \\
\hline Yes & $42(66.7)$ & 21(33.3) & & $52(82.5)$ & 11(17.5) & \\
\hline No & 197(56.3) & $153(43.7)$ & & 244(69.7) & $106(30.3)$ & \\
\hline $\begin{array}{l}\text { Presence of } \\
\text { respiratory symptoms } \\
\text { in the last } 2 \text { weeks }\end{array}$ & & & 0.158 & & & 0.046 \\
\hline
\end{tabular}




\begin{tabular}{|c|c|c|c|c|c|c|}
\hline \multirow[t]{2}{*}{ Characteristics } & \multicolumn{2}{|c|}{$\begin{array}{l}\text { Perceived efficacy of } \\
\text { COVID-19 prevention } \\
\text { methods }\end{array}$} & \multirow[t]{2}{*}{ P-value } & \multicolumn{2}{|c|}{$\begin{array}{l}\text { Intention to carry out } \\
\text { COVID- } 19 \text { prevention } \\
\text { methods }\end{array}$} & \multirow[t]{2}{*}{$\begin{array}{l}\mathrm{P} \text { - } \\
\text { value }\end{array}$} \\
\hline & $\begin{array}{l}\text { High }(\mathrm{N}= \\
239), \\
\text { n (\%) }\end{array}$ & $\begin{array}{l}\operatorname{Low}(\mathrm{N}= \\
174), \mathrm{n}(\%)\end{array}$ & & $\begin{array}{l}\operatorname{High}(\mathrm{N}= \\
296), \mathrm{n} \\
(\%)\end{array}$ & $\begin{array}{l}\operatorname{Low}(\mathrm{N}=117), \mathrm{n} \\
(\%)\end{array}$ & \\
\hline Yes & $28(68.3)$ & 13(31.7) & & $35(85.4)$ & $6(14.6)$ & \\
\hline No & 211(56.7) & $161(43.3)$ & & 261(70.2) & 111(29.8) & \\
\hline Wear face mask & & & $\begin{array}{l}< \\
0.001\end{array}$ & & & 0.014 \\
\hline Yes & $169(64.8)$ & $92(35.2)$ & & 198(75.9) & $63(24.1)$ & \\
\hline No & $70(46.1)$ & $82(53.9)$ & & $98(64.5)$ & $54(33.5)$ & \\
\hline Hand sanitizers & & & 0.036 & & & 0.013 \\
\hline Yes & $73(66.4)$ & $37(33.6)$ & & $89(80.9)$ & 21(19.1) & \\
\hline No & $166(54.8)$ & $137(45.2)$ & & 207(68.3) & $96(31.7)$ & \\
\hline Social support & & & 0.199 & & & 0.772 \\
\hline Yes & $187(56.3)$ & $145(43.7)$ & & $239(27.0)$ & $93(28.0)$ & \\
\hline No & $52(64.2)$ & $29(35.8)$ & & $57(70.4)$ & $24(29.6)$ & \\
\hline Travel history & & & 0.267 & & & 0.763 \\
\hline Yes & 12(75.0) & $4(25.0)$ & & $12(75.0)$ & $4(25.0)$ & \\
\hline No & $227(52.7)$ & $170(47.3)$ & & $284(71.5)$ & $113(28.5)$ & \\
\hline $\begin{array}{l}\text { Member of } \\
\text { community health } \\
\text { insurance }\end{array}$ & & & 0.293 & & & 0.506 \\
\hline Yes & $107(55.2)$ & $87(44.8)$ & & 136(70.1) & 58(29.9) & \\
\hline No & $132(60.3)$ & $87(39.7)$ & & $160(73.1)$ & $59(26.9)$ & \\
\hline
\end{tabular}


Table 6

Factors associated to low perceived efficacy and low intention to carry out COVID-19 prevention methods among chronic disease patients in Dessie town, northeast Ethiopia, $2020(n=413)$

\section{Characteristics}

\section{Perceived efficacy of COVID-19 Intention to carry out COVID-19 prevention methods}

\section{$\operatorname{COR}(95 \% \mathrm{Cl}) \quad \mathrm{AOR}(95 \% \mathrm{Cl}) \quad \operatorname{COR}(95 \% \mathrm{Cl}) \quad \mathrm{AOR}(95 \% \mathrm{Cl})$}

Age( year)

\begin{tabular}{|c|c|c|c|c|}
\hline $18-34$ & $\begin{array}{l}1.88(1.17- \\
3.02)\end{array}$ & $\begin{array}{l}2.48(1.42- \\
4.31)^{\star}\end{array}$ & $\begin{array}{l}1.41(0.84- \\
2.37)\end{array}$ & $\begin{array}{l}1.55(0.88- \\
2.75)\end{array}$ \\
\hline $35-54$ & $\begin{array}{l}1.08(0.66- \\
1.77)\end{array}$ & $\begin{array}{l}1.28(0.71- \\
2.31)\end{array}$ & $\begin{array}{l}1.24(0.72- \\
2.12)\end{array}$ & $\begin{array}{l}1.68(0.93- \\
3.02)\end{array}$ \\
\hline$>=55$ & 1 & 1 & 1 & 1 \\
\hline \multicolumn{5}{|l|}{ Sex } \\
\hline Male & $\begin{array}{l}2.02(1.36- \\
3.01)\end{array}$ & $\begin{array}{l}2.75(1.73- \\
4.37)^{\star}\end{array}$ & $\begin{array}{l}1.86(1.20- \\
2.87)\end{array}$ & $\begin{array}{l}2.06(1.28- \\
3.31)^{\star}\end{array}$ \\
\hline Female & 1 & 1 & 1 & 1 \\
\hline \multicolumn{5}{|c|}{ Residence } \\
\hline Urban & 1 & 1 & 1 & 1 \\
\hline Rural & $\begin{array}{l}1.91(1.26- \\
2.87)\end{array}$ & $\begin{array}{l}1.38(0.85- \\
2.24)\end{array}$ & $\begin{array}{l}2.13(1.37- \\
3.31)\end{array}$ & $\begin{array}{l}1.89(1.16- \\
3.07)^{\star}\end{array}$ \\
\hline
\end{tabular}

Educational status

No formal education

$2.69(1.31-$

3.42(1.47-

$7.94)^{\star}$

1.91(0.83-

4.37)

2.45(0.97-

5.54)

1.64(0.67-

$2.00(0.82-$

4.85)

6.18)

Primary school

1.91(0.87-

4.03)

$1.71(0.69-$

1.21(0.49-

4.21)

2.12(0.81-

4.18)

2.98)

1

5.56)

Secondary school

$\begin{array}{ll}1.26(0.56- & 1.21(0.49- \\ 2.82) & 2.98)\end{array}$

College and above

1

1

1

$2.10(0.79-$

5.58)

Duration of illness ( year)

\begin{tabular}{lllll}
$<5$ & 1 & 1 & 1 & 1 \\
\hline $5-10$ & $1.44(0.84-$ & $1.71(0.93-$ & $1.94(1.10-$ & $2.19(1.19-$ \\
& $2.48)$ & $3.13)$ & $3.42)$ & $4.01)^{\star}$ \\
$>10$ & $1.69(0.88-$ & $1.94(0.92-$ & $1.87(0.95-$ & $2.40(1.15-$ \\
& $3.23)$ & $4.07)$ & $3.67)$ & $4.98)^{\star}$
\end{tabular}




\begin{tabular}{|llll|}
\hline Characteristics & \multicolumn{2}{l}{$\begin{array}{l}\text { Perceived efficacy of COVID-19 } \\
\text { prevention methods }\end{array}$} & $\begin{array}{l}\text { Intention to carry out COVID-19 } \\
\text { prevention methods }\end{array}$ \\
\hline COR $(95 \% \mathrm{Cl})$ & $\mathrm{AOR}(95 \% \mathrm{Cl})$ & $\mathrm{COR}(95 \% \mathrm{Cl})$ & $\mathrm{AOR}(95 \% \mathrm{Cl})$
\end{tabular}

Presence of additional co-
morbidity

$\begin{array}{lllll}\text { Yes } & 1 & 1 & 1 & 1 \\ \text { No } & 1.55(0.88- & 1.88(0.94- & 2.05(1.03- & 1.86(0.85- \\ & 2.73) & 3.74) & 4.09) & 4.05)\end{array}$

Presence of respiratory

symptoms in the last 2 weeks

$\begin{array}{lllll}\text { Yes } & 1 & 1 & 1 & 1 \\ \text { No } & 1.64(0.82- & 1.57(0.69- & 2.48(1.01- & 2.53(0.94- \\ & 3.27) & 3.58) & 6.06) & 6.74)\end{array}$

Wear face mask

$\begin{array}{lllll}\text { Yes } & 1 & 1 & 1 & 1 \\ \text { No } & 2.15(1.43- & 1.64(1.03- & 1.73(1.12- & 1.38(0.86- \\ & 3.23) & 2.61)^{*} & 2.68) & 2.23)\end{array}$

Use hand sanitizers

$\begin{array}{lllll}\text { Yes } & 1 & 1 & 1 & 1 \\ \text { No } & 1.62(1.03- & 1.53(0.89- & 1.96(1.15- & 1.67(0.93- \\ & 2.56) & 2.64) & 3.35) & 3.02)\end{array}$

Have social support?

$\begin{array}{lll}\text { Yes } & 1.39(0.84- & 1.65(0.91- \\ & 2.30) & 2.98) \\ \text { No } & 1 & 1\end{array}$

*Significantly associated at $p$-value of $<0.05$,

\section{Discussion}

In Ethiopia, peoples infected with SARS-CoV-2 have been increasing significantly from day to day despite of government mitigation measures (4). As of 30 August, 2020, a total of 50,000 peoples have been infected with corona virus and 770 deaths were reported in Ethiopia (9). In the era of COVID-19 pandemic, every individual is expected to be susceptible to be infected and it brings significant morbidity and mortality. Individuals' perceived susceptibility to the novel corona virus infection and fear were the main predictor to the utilization and engagement of COVID-19 protective methods and behaviors $(48,49,55)$. A lot of factors might be contributed to the widespread distribution of the disease in Ethiopia. In our context, though not studied, one of the main reasons could be related to wrong perception of people 
towards the disease and the recommended prevention measures. Studies revealed that severity of COVID19 was found to be high in patients who had chronic disease concomitantly $(11,18)$. Thus, patients who have been living with chronic diseases should perceive as they are the most vulnerable group of peoples to COVID-19 and as much as possible they should carry out all the recommended protective measures. The more peoples perceived as the recommended COVID-19 prevention measures are effective, the more they will carry out those measures. However, in our study, $42.1 \%(95 \% \mathrm{Cl}: 37.3-46.5)$ of participants had low perception to the efficacy of COVID-19 prevention measures. Specifically, more than half of participants were perceived that wearing face mask (58.3\%) and washing hands with soap and water (61\%) helps to prevent COVID-19. This is nearly similar with studies reported in Egypt (56), India (57) and Australia (58) where more than half of respondents were perceived face mask and hand washing as an effective COVID-19 prevention measures. In the present study, staying at home (90.5\%) and selfquarantine (if suspected) (94.5\%) were the main prevention measures perceived to be effective in preventing COVID-19 pandemic than the other precautionary behaviors. However, it contradicted with a study reported in Korea that hand hygiene and wearing face mask were perceived as more effective measures (59). Our study revealed that uneducated patients were more likely to had low perception about the efficacy of COVID-19 prevention methods compared to more educated patients. This could be due to the fact that more educated peoples have more knowledge about the disease and the recommended prevention strategies. COVID-19 severe clinical disease and death have been reported mainly in peoples with advanced age and patients who had chronic disease concomitantly $(11,18)$. In our study, an independent association was observed between young adults and low perceived efficacy of COVID-19 prevention methods. Furthermore, male gender and face mask non-users were significantly associated to low perceived efficacy of recommended prevention methods.

Risk perception to COVID-19 was one of the main determinant factor to the adoption and utilization of COVID-19 prevention measures (60). In this study, $28.3 \%$ (95\% Cl: $24.1-32.7)$ of participants had low intention to carry out COVID-19 prevention measures. In Ethiopia, at the beginning of the pandemic, many people were observed to implement the adopted prevention $(43,61)$ methods. However, after three to four months of the outbreak, people's engagement in COVID-19 prevention measures and health seeking behaviors have been decreased extensively; as a result the number of people infected with the virus and related death have been increased dramatically(9). In our study, $63.2 \%$ of participants had used face mask so as to prevent themselves from corona virus infection which is similar to a study reported in South Korea (59) but lower than a study reported in Pakistan (62). However, about $81.9 \%$ of participants had an intention of wearing face mask in the present study which is higher than a study reported from Egypt(35\%) (56). In Ethiopia, numerous factors could contribute to the reduction of COVID-19 prevention measures and behaviors used in the population. Those factors may be related with lack of awareness, negligence, perception, resource limitation or a combination of those factors (61). In this study, we have also assessed the main reason why participants had no willingness to carry out the most important COVID-19 prevention measures and behaviors. As a result, "takes too much effort" was the main reason identified by participants for not willing to wear face masks and not to maintain their hand hygiene. It is mainly associated with lack of resources and study from Kenya also supported our finding; expensive 
hand sanitizers and lack of personal water source were the main barriers (63). In addition, $33.8 \%$ of participants described that they will not maintain physical distancing because they had doubt whether it helps to prevent the disease or not and $24.8 \%$ of participants will not stay home so as to prevent COVID19 because of other peoples in their environment will not carry out this measure. In this study, male patients had low intention to carry out COVID-19 prevention methods and behaviors compared to females. It is supported by study findings in Chicago (64) and Hong Kong (51) that female patients were maintaining social distancing more than males. Despite of disease severity in advanced age and in chronic disease patients $(11,18)$, multivariable analysis of this study revealed an independent association between longer duration of living with chronic disease and low intention to carry out COVID19 prevention methods.

In the present study, patients from rural area were less likely to carry out adopted COVID-19 prevention measures than urban residing patients which is in line with a study reported from China (65). This could be related to lack of information accessibility and awareness about the disease in rural resident. The possible limitation of this study could be related to the nature of cross sectional study design used and method of data collection which may predispose respondents for social desirability and recall bias. Additionally, this study was done on selected hospitals of Dessie town, thus our finding may not be generalized to the overall population of the town. Furthermore, factors related to health care setting and economical status of patients was not assessed which could have impact on adoption and utilization of COVID-19 prevention methods and behaviors.

\section{Conclusions}

In this study, significant proportion of chronic disease patients (42\%) had low perception about the efficacy of COVID-19 prevention measures and behaviors and nearly one-third of participants (28.3\%) had low intention to carry out the adopted prevention measures. our study revealed that young adults, male gender, uneducated and face mask non-users were significantly associated with low perceived efficacy of COVID-19 prevention methods. However, male gender, rural residents and patients with longer duration of chronic disease were significantly associated with low intention to carry out those prevention methods and behaviors. Thus, an intensified awareness creation program has to be provided about COVID-19 prevention measures and their risk of infection if they did not carry out those measures. Furthermore, interventions aimed to enhance patients' attitude and behavioral changes on COVID-19 prevention measures have to be provided.

\section{Abbreviations}

AOR

Adjusted Odds Ratio; Cl:Confidence Interval; COR:Crude Odds Ratio; COVID-19:Novel Corona Virus Disease 2019; SARS-CoV-2:Sever Acute Respiratory Syndrome Corona Virus 2; TB:Tuberculosis; WHO:World Health Organization; SD:Standard Deviation. 


\section{Declarations}

\section{Acknowledgments}

We would like to thank Wollo University for the ethical approval and funding this project. We also forward our thanks to study participants for their valuable information and supervisor and data collectors for their commitment.

\section{Consent to publication}

Not applicable

\section{Authors' contribution}

ADN, SGA and YGT were equally participated from conceptualization up to manuscript preparation and all authors have read and approved the final manuscript.

\section{Funding}

This project was funded by Wollo University but it has no role in manuscript preparation and publication

\section{Availability of data and materials}

All relevant data are available in the manuscript

\section{Ethical approval and consent to participate}

Ethical clearance was obtained from ethical review committee of Wollo University College of Medicine and Health Sciences with reference number of WU/324/T-01/2020. Data was collected after written consent was obtained and confidentiality of participants was maintained throughout the study by excluding their personal identifiers in the questionnaire.

\section{Competing interest- No}

\section{Author details}

${ }^{1}$ Assistant professor, Department of Nursing, School of Nursing and Midwifery, College of medicine and health sciences, Wollo University, Dessie, Ethiopia. ${ }^{2}$ Assistant professor ,Department of Community and Mental health, College of Medicine and Health Sciences, Wollo University, Dessie, Ethiopia.

\section{References}

1. WHO:. Novel Coronavirus(2019-nCoV)Situation Report-11. retrived from https://www.who.int/docs/default-source/coronaviruse/situation-reports/20200131-sitrep-11ncov.pdf?sfvrsn=de7c0f7_4. 2020. 
2. Coronavirus disease 2019 (COVID-19)Situation Report - 51. retrived from https://www.who.int/docs/default-source/coronaviruse/situation-reports/20200311-sitrep-51-covid19.pdf?sfvrsn=1ba62e57_10, (2020).

3. Clark A, Jit M, Warren-Gash C, Guthrie B, Wang HH, Mercer SW, et al. Global, regional, and national estimates of the population at increased risk of severe COVID-19 due to underlying health conditions in 2020: a modelling study. The Lancet Global Health. 2020.

4. WHO. Coronavirus disease (COVID-19) Weekly Epidemiological Update1 16August 2020.retrived from https://www.who.int/docs/default-source/coronaviruse/situation-reports/20200817-weekly-epiupdate-1.pdf?sfvrsn=b6d49a76_4. 2020.

5. Wells CR, Stearns JK, Lutumba P, Galvani AP. COVID-19 on the African continent. The Lancet Infectious Diseases. 2020.

6. Achoki T, Alam U, Were L, Gebremedhin T, Senkubuge F, Lesego A, et al. COVID-19 pandemic in the African continent: forecasts of cumulative cases, new infections, and mortality. medRxiv. 2020.

7. FMOH E. first case of covid-19 confirmed in ethiopia: avilabe at http://www.moh.gov.et/ejcc/am/node/194 2020.

8. Baye K. COVID-19 prevention measures in Ethiopia: Current realities and prospects: Intl Food Policy Res Inst; 2020.

9. WHO. Coronavirus disease (COVID-19) Weekly Epidemiological Update( 30 August 2020): avilable at https://www.who.int/docs/default-source/coronaviruse/situation-reports/20200831-weekly-epiupdate-3.pdf?sfvrsn=d7032a2a_4 2020.

10. Kobia F, Gitaka J. COVID-19: Are Africa's diagnostic challenges blunting response effectiveness? AAS Open Research. 2020;3.

11. Al-Qahtani AA. Severe Acute Respiratory Syndrome Coronavirus 2 (SARS-CoV-2): Emergence, history, basic and clinical aspects. Saudi Journal of Biological Sciences. 2020.

12. Acter T, Uddin N, Das J, Akhter A, Choudhury TR, Kim S. Evolution of severe acute respiratory syndrome coronavirus 2 (SARS-CoV-2) as coronavirus disease 2019 (COVID-19) pandemic: A global health emergency. Science of the Total Environment. 2020:138996.

13. Getaneh Y, Yizengaw A, Adane S, Zealiyas K, Abate Z, Leulseged S, et al. Global lessons and Potential strategies in combating COVID-19 pandemic in Ethiopia: Systematic Review. medRxiv. 2020.

14. Rasmussen SA, Smulian JC, Lednicky JA, Wen TS, Jamieson DJ. Coronavirus Disease 2019 (COVID19) and Pregnancy: What obstetricians need to know. American journal of obstetrics and gynecology. 2020.

15. Gulati A, Pomeranz C, Qamar Z, Thomas S, Frisch D, George G, et al. A Comprehensive Review of Manifestations of Novel Coronaviruses in the Context of Deadly COVID-19 Global Pandemic. The American Journal of the Medical Sciences. 2020.

16. Lai C-C, Liu YH, Wang C-Y, Wang Y-H, Hsueh S-C, Yen M-Y, et al. Asymptomatic carrier state, acute respiratory disease, and pneumonia due to severe acute respiratory syndrome coronavirus 2 
(SARSCoV-2): facts and myths. Journal of Microbiology, Immunology and Infection. 2020.

17. De Felice FG, Tovar-Moll F, Moll J, Munoz DP, Ferreira ST. Severe Acute Respiratory Syndrome Coronavirus 2 (SARS-CoV-2) and the Central Nervous System. Trends in neurosciences. 2020.

18. Li J, He X, Zhang W, Li X, Zhang Y, Li S, et al. Meta-analysis investigating the relationship between clinical features, outcomes, and severity of severe acute respiratory syndrome coronavirus 2 (SARSCoV-2) pneumonia. American Journal of Infection Control. 2020.

19. Ortiz-Prado E, Simbaña-Rivera K, Gómez-Barreno L, Rubio-Neira M, Guaman LP, Kyriakidis NC, et al. Clinical, molecular and epidemiological characterization of the SARS-CoV2 virus and the Coronavirus disease 2019 (COVID-19), a comprehensive literature review. Diagnostic Microbiology and Infectious Disease. 2020:115094.

20. Stokes EK, Zambrano LD, Anderson KN, Marder EP, Raz KM, Felix SEB, et al. Coronavirus Disease 2019 Case Surveillance-United States, January 22-May 30, 2020. Morbidity and Mortality Weekly Report. 2020;69(24):759.

21. Global H. AIDS statistics: 2019 Factsheet. UNAIDS. 2019.

22. Girum T, Mesfin D, Bedewi J, Shewangizaw M. The Burden of Noncommunicable Diseases in Ethiopia, 2000-2016: Analysis of Evidence from Global Burden of Disease Study 2016 and Global Health Estimates 2016. International Journal of Chronic Diseases. 2020;2020.

23. Annabel B, Anna D, Hannah M. Global tuberculosis report 2019. Geneva: World Health Organization. 2019.

24. Organization WH. Country \& technical guidance-Coronavirus disease (COVID-19). Geneva, Switzerland: WHO. 2020.

25. Bong C-L, Brasher C, Chikumba E, McDougall R, Mellin-Olsen J, Enright A. The COVID-19 Pandemic: Effects on Low-and Middle-Income Countries. Anesthesia and analgesia. 2020.

26. Kanu IA. COVID-19 and the economy: an African perspective. Journal of African Studies and Sustainable Development. 2020;3(2).

27. Ataguba JE. COVID-19 pandemic, a war to be won: understanding its economic implications for Africa. Springer; 2020.

28. Lone SA, Ahmad A. COVID-19 pandemic-An African perspective. Emerging Microbes \& Infections. 2020:1-28.

29. Rosenthal PJ, Breman JG, Djimde AA, John CC, Kamya MR, Leke RG, et al. COVID-19: shining the light on Africa. The American Journal of Tropical Medicine and Hygiene. 2020;102(6):1145.

30. Zhou S-J, Zhang L-G, Wang L-L, Guo Z-C, Wang J-Q, Chen J-C, et al. Prevalence and sociodemographic correlates of psychological health problems in Chinese adolescents during the outbreak of COVID-19. European Child \& Adolescent Psychiatry. 2020:1-10.

31. Yusuf S. POLICY BRIEFCOVID-19 and political stability in Ethiopia ISS. 2020.

32. Davies NG, Sweeney S, Torres-Rueda S, Bozzani F, Kitson N, Barasa E, et al. The impact of Coronavirus disease 2019 (COVID-19) on health systems and household resources in Africa and 
South Asia. medRxiv. 2020.

33. Paintsil E. COVID-19 threatens health systems in sub-Saharan Africa: the eye of the crocodile. The journal of clinical investigation. 2020;130(6).

34. Blanton RE, Mock NB, Hiruy HN, Schieffelin JS, Doumbia S, Happi C, et al. African Resources and the Promise of Resilience against COVID-19. The American Society of Tropical Medicine and Hygiene; 2020.

35. Geda A. The Macroeconomic and Social Impact of COVID-19 in Ethiopia and Suggested Direction for Policy Response. Addis Ababa: Addis Ababa University; 2020.

36. Mulugeta M. The Impact of COVID-19 Pandemic on Food Security in Ethiopia.

37. Hirvonen K, Abate GT, de Brauw A. Food and nutrition security in Addis Ababa, Ethiopia during COVID-19 pandemic: May 2020 report: Intl Food Policy Res Inst; 2020.

38. Nakkazi E. Obstacles to COVID-19 control in east Africa. The Lancet Infectious Diseases. 2020;20(6):660.

39. Basnet S, Adhikari N, Koirala J. Challenges in setting up pediatric and neonatal intensive care units in a resource-limited country. Pediatrics. 2011;128(4):e986-e92.

40. Ali I, Alharbi OM. COVID-19: Disease, management, treatment, and social impact. Science of the Total Environment. 2020:138861.

41. Tenforde MW, Rose EB, Lindsell CJ, Shapiro NI, Files DC, Gibbs KW, et al. Characteristics of Adult Outpatients and Inpatients with COVID-19-11 Academic Medical Centers, United States, March-May 2020. Morbidity and Mortality Weekly Report. 2020;69(26):841.

42. Organization WH. Rational use of personal protective equipment for coronavirus disease (COVID-19): interim guidance, 27 February 2020. World Health Organization, 2020.

43. Bekele D, Tolossa T, Tsegaye R, Teshome W. The knowledge and practice towards COVID-19 pandemic prevention among residents of Ethiopia. An online cross-sectional study. BioRxiv. 2020.

44. Dagne H, Alemu KA, Dagnew B, Taddesse D, Alemayehu AM, Andualem Z, et al. Prevention Practice and Associated Factors of Coronavirus disease 2019 (COVID-19) Outbreak among Educated Ethiopians: An online Based Cross-sectional Survey. 2020.

45. Akalu Y, Ayelign B, Molla MD. Knowledge, attitude and practice towards COVID-19 among chronic disease patients at Addis Zemen Hospital, Northwest Ethiopia. Infection and drug resistance. 2020;13:1949.

46. Nigussie TF, Azmach NN. knowledge, attitude and practice towards covid-19 among arba minch town, southern ethiopia. GSJ. 2020;8(6).

47. Mola S, Aweke Z, Jemal B, Hussein R, Hailu S, Neme D, et al. Magnitude and associated factors for attitude and practice of Southern Ethiopian residents toward COVID-19 and its preventions: A community based cross sectional study. 2020.

48. Wise T, Zbozinek TD, Michelini G, Hagan CC. Changes in risk perception and protective behavior during the first week of the COVID-19 pandemic in the United States. 2020. 
49. Duan T, Jiang H, Deng X, Zhang Q, Wang F. Government intervention, risk perception, and the adoption of protective action recommendations: Evidence from the COVID-19 prevention and control experience of China. International Journal of Environmental Research and Public Health. 2020;17(10):3387.

50. EFDR, Population census commision. Summary and Statistical Report of the 2007 Population and Housing Census Results. 2008.

51. Kwok KO, Li KK, Chan HH, Yi YY, Tang A, Wei WI, et al. Community responses during the early phase of the COVID-19 epidemic in Hong Kong: risk perception, information exposure and preventive measures. MedRxiv. 2020.

52. Nasir EF, Almahdi HA. Study of the Sudanese perceptions of COVID-19: Applying the Health Belief Model. medRxiv. 2020.

53. Wadood MA, Lee LL, Huq MM, Mamun A, Mohd S, Hossain MG. Practice and perception of Bangladeshi adults toward COVID-19: a cross-sectional study. 2020.

54. ECOM, effective comminication in outbreak management for Europe, Standard questionnaire on risk perception of an infectious disease outbreak: retrived from http://ecomeu.info/wpcontent/uploads/2015/11/Standard-questionnaire-risk-perception-ECOM-november-2015.pdf. 2015.

55. Yıldııım M, Geçer E, Akgül Ö. The impacts of vulnerability, perceived risk, and fear on preventive behaviours against COVID-19. Psychology, Health \& Medicine. 2020:1-9.

56. Abdelhafiz AS, Mohammed Z, Ibrahim ME, Ziady HH, Alorabi M, Ayyad M, et al. Knowledge, perceptions, and attitude of egyptians towards the novel coronavirus disease (COVID-19). Journal of Community Health. 2020:1-10.

57. Nazli T, Raheem A, Kishore J. Perceptions and Practices of the Adult Population in Response to SARS-CoV-2 Pandemic in India. Epidemiology International (E-ISSN: 2455-7048). 2020;5(2):10-6.

58. Seale H, Heywood AE, Leask J, Steel M, Thomas S, Durrheim DN, et al. COVID-19 is rapidly changing: Examining public perceptions and behaviors in response to this evolving pandemic. medRxiv. 2020.

59. Lee M, You M. Psychological and behavioral responses in South Korea during the early stages of coronavirus disease 2019 (COVID-19). International Journal of Environmental Research and Public Health. 2020;17(9):2977.

60. Ahmad M, Iram K, Jabeen G. Perception-based influence factors of intention to adopt COVID-19 epidemic prevention in China. Environmental research. 2020:109995.

61. Kebede Y, Yitayih Y, Birhanu Z, Mekonen S, Ambelu A. Knowledge, perceptions and preventive practices towards COVID-19 early in the outbreak among Jimma university medical center visitors, Southwest Ethiopia. PloS one. 2020;15(5):e0233744.

62. Rizwan W, Sadiq M, Mushtaq A, Lodhi AM, Awan ZR, Rana MN. Knowledge, Risk Perception and Behavioral Response of COVID-19 Among the General Population Attending Children's Hospital, Lahore. Annals of King Edward Medical University. 2020;26(Special Issue):276-83.

63. Austrian K, Pinchoff J, Tidwell JB, White C, Abuya T, Kangwana B, et al. Preprint-COVID-19 related knowledge, attitudes, practices and needs of households in informal settlements in Nairobi, Kenya. 
2020.

64. O'Conor R, Opsasnick L, Benavente JY, Russell AM, Wismer G, Eifler M, et al. Knowledge and Behaviors of Adults with Underlying Health Conditions During the Onset of the COVID-19 US Outbreak: The Chicago COVID-19 Comorbidities Survey. Journal of community health. 2020:1-9.

65. Chen X, Chen H. Differences in Preventive Behaviors of COVID-19 between Urban and Rural Residents: Lessons Learned from A Cross-Sectional Study in China. International journal of environmental research and public health. 2020;17(12):4437. 